\title{
Jet Quenching with ATLAS and CMS
}

\author{
Pelin Kurt $^{1, \mathrm{a}}$ for the ATLAS and CMS Collaborations \\ ${ }^{1}$ University of Illinois at Chicago, USA
}

\begin{abstract}
An overview of the most recent results on jet quenching physics obtained using $\mathrm{PbPb}$ collision data collected with the ATLAS and the CMS experiments at $\sqrt{s_{\mathrm{NN}}}=2.76$ $\mathrm{TeV}$ will be presented. These measurements make use of many different observables, including momentum imbalance of dijet and photon-jet events, nuclear modification factors $R_{\mathrm{AA}}$ and $R_{\mathrm{CP}}$, as well as jet fragmentation functions, jet shapes, and the flavor dependence of jet quenching. The measurements in $\mathrm{PbPb}$ collisions will be compared to those obtained from pp collisions at the same center-of-mass energy. The effects of the parton energy loss in the hot and dense medium probed with the different observables will be discussed.
\end{abstract}

\section{Introduction}

Heavy ion collisions at the Large Hadron Collider (LHC) allow one to study the phases of nuclear matter predicted by the theory of the strong interaction Quantum Chromodynamics (QCD) [1,2]. Jets associated with the hard scattering of partons are a powerful probe of the hot, dense matter created in heavy-ion collisions. This medium is commonly referred to as a Quark-Gluon Plasma (QGP). The partons lose energy while traversing the medium via elastic processes (collisional parton energy loss) or inelastic processes (radiative parton energy loss) [3]. At RHIC, indirect measurements of energy loss in the medium ("jet quenching") have been made by studying high momentum jet fragmentation products [4, 5]. More recently, the ATLAS [6] and the Compact Muon Solenoid (CMS) [7] detectors have been used to study for parton energy loss in the quark-gluon plasma with leading particle and jet coincidence measurements. The ATLAS and the CMS detectors are general purpose particle experiments operating at the LHC. These detectors are built to be sensitive to a wide-variety of physics processes, and are well suited for the study of heavy-ion collisions. We present some selected measurements related to parton energy loss in $\mathrm{PbPb}$ collisions at a nucleon-nucleon center-of-mass energy of $\sqrt{s_{\mathrm{NN}}}=2.76 \mathrm{TeV}$ collected in 2010 and 2011 using the ATLAS and CMS detectors.

\section{Experimental Techniques}

The heavy-ion analyses at ATLAS and CMS share some common experimental methods. In these measurements it is common to first perform a measurement where the medium is present, and then

ae-mail: pkurt@cern.ch 
to make the same measurement in pp or pp-like collisions, and finally compare to study the mediuminduced modifications. For this comparison, a pp reference data (at the same center-of-mass energy) was collected at the LHC.

The capabilities of the ATLAS and CMS detectors allow us to investigate various hard probes, using excellent tracking, calorimetric, and muon systems which cover a large range in pseudorapidity. All of these detectors have sufficient granularity and resolution to function well even in the highest multiplicities encountered in $\mathrm{PbPb}$ collisions.

It is also important to note that heavy ions are extended objects, so the impact parameter is an important characterization of the events. The centrality of the collisions is defined as a fraction of the total nucleus-nucleus inelastic cross section, with $0 \%$ denoting the most central collisions with impact parameter 0 , and $100 \%$ - the most peripheral collisions. In these analyses, centrality was determined from minimum bias events based on the total energy from both forward hadronic calorimeters [8, 9]. The more frequent peripheral events with a large impact parameter produce very few particles, while the central ones with a small impact parameter produce many more particles because of the increased number of nucleon-nucleon interactions.

\section{Results}

In contrast to pp collisions, a large fraction of imbalanced dijet transverse momentum has been observed in $\mathrm{PbPb}$ collisions at $\sqrt{s_{\mathrm{NN}}}=2.76 \mathrm{TeV}$. This observation was first reported by ATLAS [8] using a data sample corresponding to an integrated luminosity of $1.7 \mu b^{-1}$, where the dijet momentum balance has been quantified in different centrality bins using an asymmetry ratio $A_{j}=\frac{E_{T_{1}}-E_{T_{2}}}{E_{T_{1}}+E_{T_{2}}}$, where $E_{T_{1}}$ is the transverse energy of the leading jet and required to be $E_{T_{1}}>100 \mathrm{GeV}$, and $E_{T_{2}}$ is the transverse energy of the subleading jet in the opposite hemisphere with $E_{T_{2}}>25 \mathrm{GeV}$. In Fig. 1 the top panel shows that the dijet asymmetry in peripheral $\mathrm{PbPb}$ events is similar to that in both pp and simulated events. However, as the events become more central, the $\mathrm{PbPb}$ data distributions develop different characteristics, indicating an increased rate of highly asymmetric dijet events. In the bottom panel, the azimuthal-angle between the dijet events are shown for different centrality classes. It is clear that the leading and subleading jets are primarily back-to-back in all centrality bins. However, a systematic increase is observed in the rate of second jets at large angles relative to the recoil direction as the events become more central. This is due to the jet-quenching effect which can cause some jets to become lower in $p_{T}$ than jets from a second hard scattering. This can result in an association of a leading jet to a jet from a different hard scattering instead of the proper rebound jet. As expected, this effect is quite visible in data where the medium effect is present.

In a more detailed study of the parton energy loss mechanism [9], CMS has investigated the redistribution of the quenched jet energy using the transverse momentum balance of charged tracks projected onto the direction of the leading jet axis, defined as $p_{T}^{\|}=\sum_{\mathrm{i}}-p_{T}^{\mathrm{i}} \cos \left(\phi_{\mathrm{i}}-\phi_{\text {Leading Jet }}\right)$, where the sum is evaluated over all tracks with $p_{T}>0.5 \mathrm{GeV} / \mathrm{c}$ and $|\eta|<2.4$. The results were then averaged over the event ensemble to obtain $\left\langle p_{T}^{\|}\right\rangle$. No explicit background subtraction is applied in this method, as the heavy-ion underlying event is not expected to give a net $p_{T}$ contribution along the leading jet axis. Fig. 2 (left) shows $\left\langle p_{T}^{\|}\right\rangle$as a function of $A_{J}$ for the most central $\mathrm{PbPb}$ collisions $(0-30 \%)$. Even for events with a very unbalanced dijet (large $A_{J}$ values), the total summed projected momentum of all the included tracks (solid circles) is close to zero. The colored bands (with vertical bars for statistical uncertainties) show the summed momentum for tracks restricted to specific $p_{T}$ ranges. The sum for tracks with $p_{T}>8 \mathrm{GeV} / \mathrm{c}$ is strongly negative, indicating that they carry excess momentum in the direction of the leading jet. This negative excess is balanced by the almost equally strong positive contribution of tracks in the $0.5 \mathrm{GeV} / \mathrm{c}$ regions. Tracks in the intermediate range, $2<p_{T}<8 \mathrm{GeV} / \mathrm{c}$, 

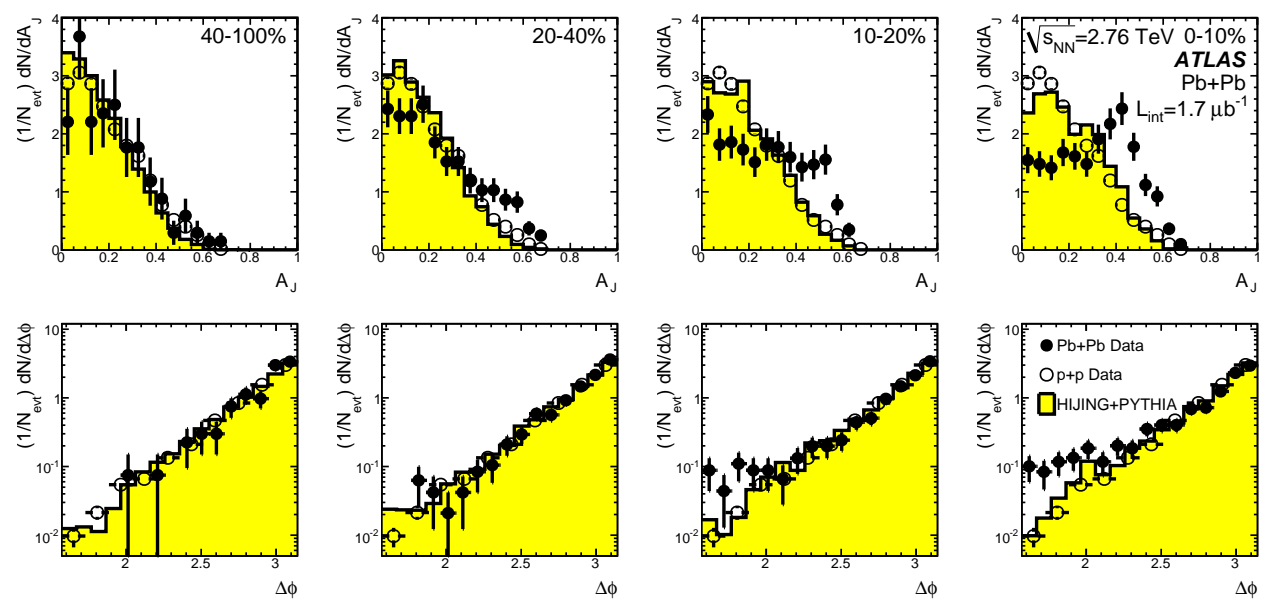

Figure 1. (top) Dijet asymmetry distributions for data (points) and unquenched heavy ion simulation with superimposed PYTHIA pp dijets (solid yellow histograms), as a function of collision centrality (left to right from peripheral to central events). Proton-proton data from $\sqrt{s_{\mathrm{NN}}}=7 \mathrm{TeV}$, analyzed with the same jet selection, is shown as open circles. (bottom) Distribution of $\Delta \phi$, the azimuthal angle between the two jets, for data and heavy ion simulation, also as a function of centrality [8].

also tend to carry excess momentum in the direction of the subleading jet, but to a lesser degree. The main conclusion here is that a large fraction of the momentum balance of the jets in unbalanced events is carried by low- $p_{T}$ particles at large radial distance to the jet axis [9].

While studies using dijets benefit from the large dijet production cross section, the energy loss of both partons makes the determination of the amount of energy lost by each parton more difficult. Correlations between isolated photons and jets have been proposed in the literature as the "golden channel" to study jet energy loss. This is because the photon retains the kinematic information of the hard scattering since it is not expected to interact with the medium. In addition, the energy resolution for photons is better, making the photon an ideal object against which to compare the jet. In order to quantify any angular broadening, the $\mathrm{PbPb}$ data were compared to both pp data and a PYTHIA+HYDJET reference which included the effect of the underlying $\mathrm{PbPb}$ event but no parton energy loss. Similar to dijet events, no angular broadening was observed beyond that seen in the pp data and MC reference at all centralities. Further details about the measurement of isolated-photon+jet correlations in $\sqrt{s_{\mathrm{NN}}}=$ 2.76 TeV pp and PbPb collisions with CMS and ATLAS can be found elsewhere [10, 11].

Energy loss of the parent partons in the medium may reduce or "suppress" the production of jets at a given transverse momentum. Such energy loss is expected to increase with medium temperature and with increasing path length of the parton in the medium [12]. As a result, there should be more suppression in "central" $\mathrm{Pb}+\mathrm{Pb}$ collisions, which have nearly complete overlap between the incident nuclei, and little or no suppression in "peripheral" collisions where the nuclei barely overlap. The jet suppression may be quantified using the central-to-peripheral ratio, $R_{\mathrm{CP}}$, the ratio of the per-event jet yields divided by the number of nucleon-nucleon collisions in a given centrality bin to the same quantity in a peripheral centrality bin formulated as:

$$
R_{\mathrm{CP}}=\frac{\left(d N / d p_{\mathrm{T}}\right) /\left\langle N_{\text {coll }}\right\rangle[\text { central }]}{\left(d N / d p_{\mathrm{T}}\right) /\left\langle N_{\text {coll }}\right\rangle[60-80 \%]},
$$




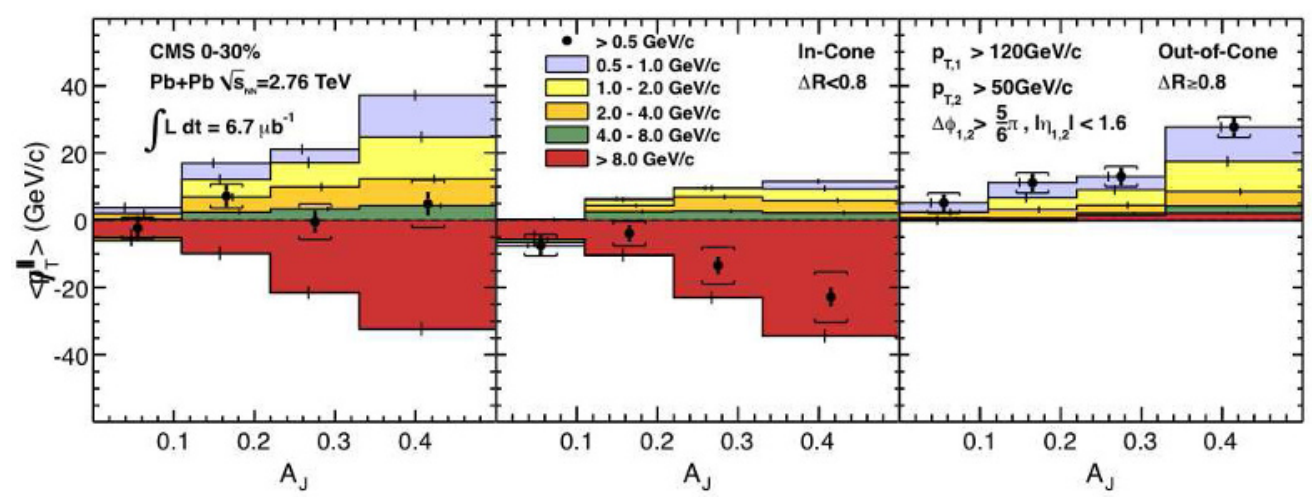

Figure 2. (Left) Average missing transverse momentum, $\left\langle p_{T}^{\|}\right\rangle$, for tracks with $p_{T}>0.5 \mathrm{GeV} / \mathrm{c}$, projected onto the leading jet axis (solid circles). The $\left\langle p_{T}^{\|}\right\rangle$values are shown as a function of dijet asymmetry for the $30 \%$ most central events. (Middle) The $\left\langle p_{T}^{\|}\right\rangle$values as a function of $A_{J}$ inside $(\Delta R<0.8)$ one of the leading or subleading jet cones. (Right) $\left\langle p_{T}^{\|}\right\rangle$outside $(\Delta R>0.8)$ the leading and subleading jet cones. For the solid circles, vertical bars and brackets represent the statistical and systematic uncertainties, respectively. Colored bands, with vertical bars for statistical uncertainties, show the contribution to $\left\langle p_{T}^{\|}\right\rangle$for five ranges of track $p_{\mathrm{T}}[9]$.

where the $\left\langle N_{\text {coll }}\right\rangle$ is the average number of nucleon-nucleon collisions occurring in heavy-ion (AA) collisions, calculated with a Glauber model with a detailed description of the nuclear collision geometry [13]. This measurement was performed by both ATLAS and the CMS experiments. An unfolding procedure is also applied to account for the effects of detector resolution. The reconstructed $E_{\mathrm{T}}$ spectrum in each centrality bin is unfolded to the particle level, taking into account the migration between bins that arises due to experimental jet energy resolution. Results are shown for the ATLAS experiment [14] in Fig. 3. This figure shows the unfolded $R_{\mathrm{CP}}$ values obtained for $\mathrm{R}=0.2$ and $\mathrm{R}=0.4$ jets (reconstructed with the anti- $k_{\mathrm{T}}$ algorithm) as a function of the jet $p_{\mathrm{T}}$ in four bins of collision centrality. The $R_{\mathrm{CP}}$ values for all centralities and for both jet radii are observed to have at most a weak variation with $p_{\mathrm{T}}$. For the $0-10 \%$ centrality bin the $R_{\mathrm{CP}}$ values for both jet radii show a factor of about two suppression in the $1 /\left\langle N_{\text {coll }}\right\rangle$ scaled jet yield. For more peripheral collisions, $R_{\mathrm{CP}}$ increases at all jet $p_{\mathrm{T}}$ relative to central collisions, with the $R_{\mathrm{CP}}$ values reaching 0.9 for the $50-60 \%$ centrality bin.

Another interesting analysis is a measurement of the jet flavor dependence of the jet quenching, which is expected to depend on the flavor of the initial parton. Gluon jets are expected to be quenched more strongly than light quark jets due to the larger color factor for gluon emission from gluons than from quarks. On the other hand, jets initiated by heavy quarks, particularly bottom quarks, are expected to radiate less than light ones. To measure this flavour dependence, the CMS collaboration has applied a b-jet identification algorithm for the first time in heavy ion collisions to perform such a measurement [15]. The purity of b-jet tagging is determined from template fits to the secondary vertex invariant mass distribution, and the efficiency of the secondary vertex tagging is estimated in a data- driven technique. The fraction of b-jet among inclusive jets is measured as a function of transverse momentum after purity and efficiency corrections in the range of $80<p_{\mathrm{T}}^{\text {jet }}<200 \mathrm{GeV} / \mathrm{c}$. The fraction of $\mathrm{b}$-jets in $\mathrm{pp}$ and $\mathrm{PbPb}$ collisions are comparable, with no $p_{\mathrm{T}}$ dependence, indicating that b-quark jets are quenched similar to the light quark jets, i.e. the $R_{\mathrm{AA}}$ value is $\approx 0.5$. These measurements have significant statistical uncertainties at present. The addition of more statistics, 

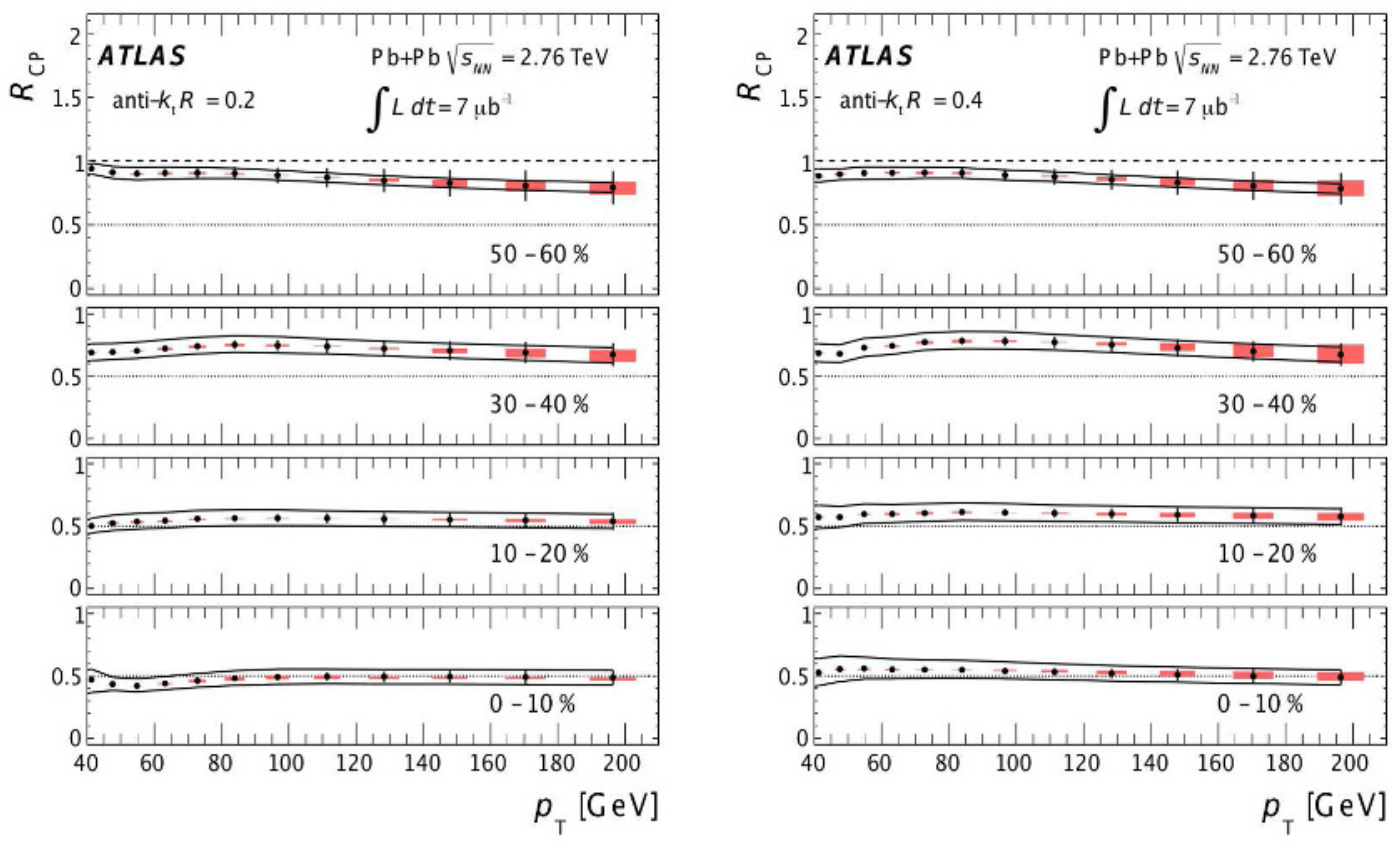

Figure 3. Unfolded $R_{\mathrm{CP}}$ values as a function of jet $p_{\mathrm{T}}$ for $\mathrm{R}=0.2$ (left) and $\mathrm{R}=0.4$ (right) anti- $k_{\mathrm{T}}$ jets in four bins of collision centrality [12]. The error bars indicate statistical errors from the unfolding, and the shaded boxes indicate unfolding regularization systematic errors that are partially correlated between points. The solid lines indicate systematic errors that are fully correlated between all points. The horizontal width of the systematic error band is chosen for presentation purposes only. Dotted lines indicate $R_{\mathrm{CP}}=0.5$, and the dashed lines on the top panels indicate $R_{\mathrm{CP}}=1$.

along with a more precise calibration of tagging efficiencies and fit template shapes, may lead to significant improvements in precision in the future.

A differential measurement has been performed to test the effects of the medium using a jet shape observable at the CMS experiment. The jet shapes are a sensitive tool for the characterization of the parton-medium interactions by utilizing the energy flow inside the jet. Predictions have been made that the jet shapes will become wider due to quenching effects [16]. We present the first experimental test of this prediction. For this measurement, jets are reconstructed using the anti- $k_{\mathrm{T}}$ jet clustering algorithm [17], with a resolution parameter $\mathrm{R}=0.3$ for both $\mathrm{PbPb}$ and pp collisions at $2.76 \mathrm{TeV}$. Individually calibrated particle candidates are used as inputs to the jet clustering algorithm. These particle candidates are reconstructed using the CMS particle flow (PF) algorithm [18]. The jet shape is defined as the average fraction of the jet transverse momentum within a cone of a given size $r$ around the jet axis. The jet shapes can be studied by using an integrated or a differential distribution. The shapes are defined as the average fraction of the transverse momentum contained inside an annulus of an inner radius $r_{\mathrm{a}}=r-\delta r / 2$ and an outer radius $r_{\mathrm{b}}=r+\delta r / 2$ as specified in the following equation

$$
\rho(r)=\frac{1}{\delta r} \frac{\sum_{r_{\mathrm{a}}<r_{i}<r_{\mathrm{b}}} p_{\mathrm{T}, i}}{\sum_{r_{i}<R} p_{\mathrm{T}, i}},
$$




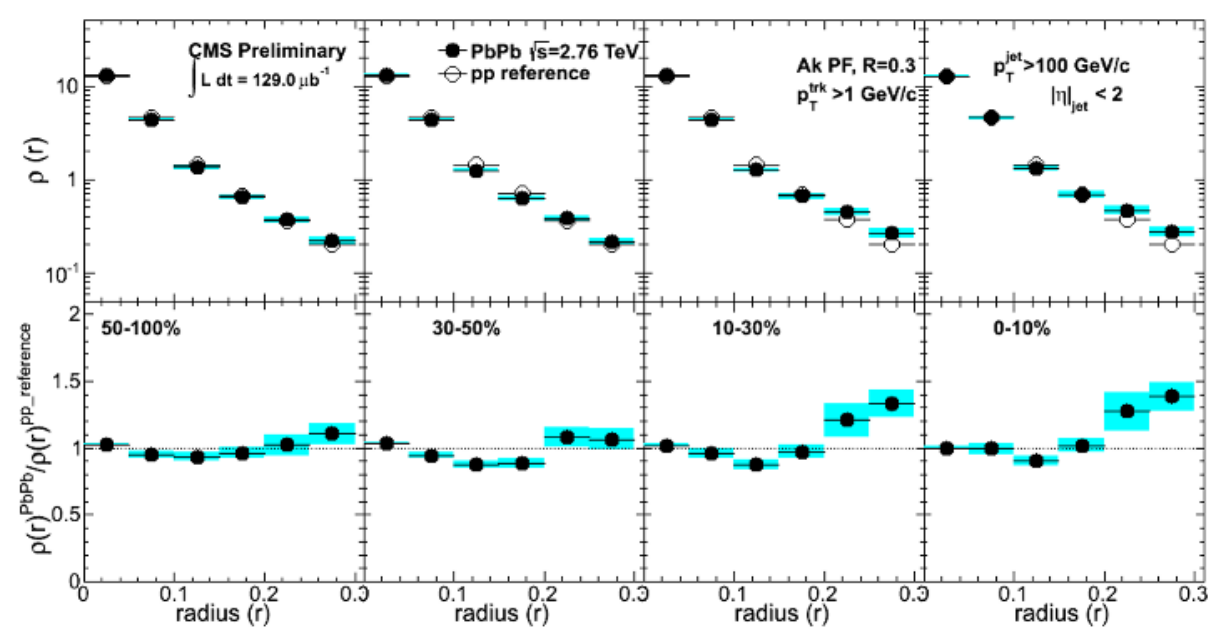

Figure 4. Differential jet shapes in $\mathrm{PbPb}$ and pp collisions are presented for different centrality bins for $p_{\mathrm{T}}^{\text {jet }}>$ $100 \mathrm{GeV} / \mathrm{c}$ with track $p_{\mathrm{T}}>1 \mathrm{GeV}$ are shown in the top panels. Results from data are shown as black points while the open circles show the reference pp. In the bottom row, the ratio of the $\mathrm{PbPb}$ and $\mathrm{pp}$ jet shapes is shown. The blue band shows the total systematic uncertainty while the error bars indicate the statistical errors [19].

where $\delta r$ is used as the annulus size, which is 0.05 . The sums run over the reconstructed particles, with the distance $r_{i}=\sqrt{\left(\eta_{i}-\eta_{j e t}\right)^{2}+\left(\phi_{i}-\phi_{j e t}\right)^{2}}$ relative to the jet axis described by $\eta_{j e t}, \phi_{j e t}$, and $R$.

A small cone size $(\mathrm{R}=0.3)$ was used for the jet reconstruction in order to suppress the underlyingevent contribution in the high multiplicity $\mathrm{PbPb}$ environment. All charged particles that pass a $p_{\mathrm{T}}>$ $1 \mathrm{GeV} / \mathrm{c}$ threshold are used to reconstruct jet shapes. Corrections for the tracking inefficiency are applied. The hot-and-dense medium is expected to modify a measured jet shape in two ways. First, the partons that fragment into jets interact with the medium directly. Secondly, the soft particle production from the underlying event adds many extra particles to the jet, predominantly at low momentum. This latter effect produces a background that must be subtracted. In order to subtract the heavyion background, an $\eta$-reflection technique [19] was used. In order to understand the medium-parton interactions we compare the $\mathrm{PbPb}$ jet shapes results with those obtained from a pp reference. For a direct comparison between $\mathrm{pp}$ and $\mathrm{PbPb}$ collisions, the jet momentum resolution deterioration in $\mathrm{PbPb}$ events is taken into account. For this purpose, the reconstructed $p_{\mathrm{T}}$ of every jet in the pp data is smeared by the quadratic difference of the jet energy resolution obtained in $\mathrm{PbPb}$ and $\mathrm{pp}$. The resulting jet $p_{\mathrm{T}}$ spectrum is compared to the spectra in $\mathrm{PbPb}$ collisions of different centrality to determine a $p_{\mathrm{T}^{-}}$ dependent weight, which is applied on a jet-by-jet basis to obtain reference jet-shape distributions. This process is needed to ensure that the comparison is free of detector effects and that the kinematic range of the jets included in the comparison is the same.

The measured differential jet shapes for $\mathrm{PbPb}$ and pp reference data are presented in Fig. 4 for different centrality bins, ranging from most-peripheral (50-100\%) to most central (0-10\%). The bottom panel shows the ratio of the $\mathrm{PbPb}$ jet shapes to the jet shapes for a pp reference obtained for the respective selections. Deviations from unity indicate modification of jet structure in the nuclear medium. We note that the jet shape spectra are normalized to unity. As a result, an excess at one distance $r$ from the jet axis has to be compensated by a depletion in another region. In all centrality classes, the ratios have a concave shape, which is more pronounced in the more central collisions. In central collisions (10-30\% and $0-10 \%$ ), an excess at large radius $r>0.2$ emerges, indicating a moderate broadening of the jets in the medium. This result is consistent with previous studies in CMS which find that the energy that the jets lose in the medium is redistributed at large distances from the jet axis outside the jet cone [9]. 


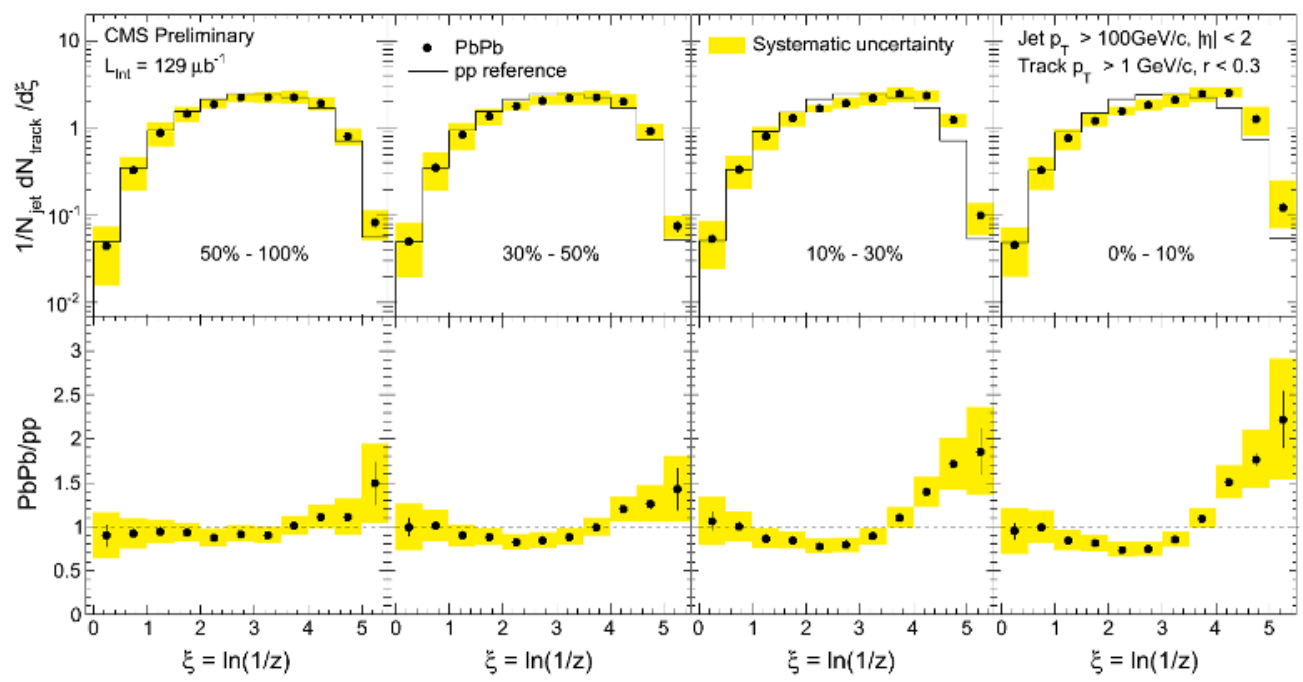

Figure 5. The top row shows the jet fragmentation functions in $\mathrm{PbPb}$ in bins of increasing centrality, overlaid with $\mathrm{pp}$. Jets are required to have $p_{\mathrm{T}}$ above $100 \mathrm{GeV} / \mathrm{c}$, and tracks to have $p_{\mathrm{T}}$ above $1 \mathrm{GeV} / \mathrm{c}$. The $\mathrm{PbPb}$ data is shown in the top row in four increasing centrality bins from left to right. The bottom row shows the ratio of each $\mathrm{PbPb}$ fragmentation function to its pp reference [19].

In another differential CMS measurement the fragmentation functions, defined as the distribution of the fraction of the jet momentum carried by tracks of different $p_{\mathrm{T}}$, are determined [19]. The fragmentation function is formulated with $\xi$, defined as $\xi=\ln \frac{1}{z} ; \mathrm{z}=\frac{p_{\|}^{\text {track }}}{p_{\mathrm{T}}^{\text {jet }}}$ where $p_{\|}^{\text {track }}$ is the momentum component of the track along the jet axis, and $p_{\mathrm{T}}^{\text {jet }}$ is the transverse momentum of the reconstructed jet, respectively. The event selection and analysis procedure are the same as in inclusive jet shapes analysis. In order to quantify the medium-related effects, the results are compared to the references based on pp data. The upper panel of Fig. 5 shows the reconstructed fragmentation functions in pp and $\mathrm{PbPb}$ data. The bottom panel shows that the modification of the fragmentation function of jets grows with the collision centrality. In the $50-100 \%$ bin, the ratio of $\mathrm{PbPb} / \mathrm{pp}$ is flat at unity which means no modification. However, an excess in high $\xi$ is observed for more central events. This implies that for central collisions the spectrum of particles in a jet has an enhanced contribution of soft particles compared to one from pp collisions.

\section{Conclusions}

The ATLAS and CMS collaborations have performed many interesting measurements in $\mathrm{PbPb}$ collisions. All these measurements in $\mathrm{PbPb}$ collisions are presented and compared with observations in 2.76 TeV pp collisions to probe for distortions from energy loss in the hot and dense medium. In summary, the fraction of dijets in which the momentum of the leading and subleading jets are significantly unbalanced rises dramatically for more central $\mathrm{PbPb}$ collisions, consistent with significant quenching of hard partons. A large fraction of the momentum "missing" from the lower energy jet is carried by low- $p_{\mathrm{T}}$ particles at relatively large angles with respect to the jet axis. The photon-jet 
correlation studies support the dijet quenching picture further. Measurement of $b$-jets shows that the heavy quark jets are quenched similar to light quark jets, with no strong dependence on jet $p_{\mathrm{T}}$. A clear centrality dependent modification of the inclusive jet rates, shapes and fragmentation function in $\mathrm{PbPb}$ collisions is now revealed. Since many of these observables have low correlation to one-another they serve as useful independent confirmations of the quenching properties, and indicate a consistent view of the hot and dense medium.

\section{References}

[1] D. A. Appel, "Jets as a probe of quark-gluon plasmas", Phys. Rev. D33 (1986) 717. doi:10.1103/PhysRevD.33.717.

[2] J. P. Blaizot and L. D. McLerran, "Jets in Expanding Quark - Gluon Plasmas", Phys. Rev. D34 (1986) 2739. doi:10.1103/PhysRevD.34.2739.

[3] J. D. Bjorken, "Energy loss of energetic partons in QGP: possible extinction of high $p_{T}$ jets in hadron-hadron collisions", (1982). FERMILAB-PUB-82-059-THY.

[4] PHENIX Collaboration, "Suppression of Hadrons with Large Transverse Momentum in Central $\mathrm{Au}+\mathrm{Au}$ Collisions at $130 \mathrm{GeV}$ ", Phys. Rev. Lett. 88, 022301 (2001).

[5] STAR Collaboration, "Direct observation of dijets in central Au+Au collisions at $200 \mathrm{GeV}$ ", Phys.Rev.Lett. 97 (2006) 162301.

[6] ATLAS Collaboration, "The ATLAS experiment at the CERN LHC", JINST 3 (2008) S08003.

[7] CMS Collaboration, "The CMS experiment at the CERN LHC", JINST 3 (2008) S08004.

[8] ATLAS Collaboration, "Observation of a Centrality-Dependent Dijet Asymmetry in Lead-Lead Collisions at $\sqrt{s_{N N}}=2.76 \mathrm{TeV}$ with the ATLAS Detector at the LHC", Phys. Rev. Lett. 105 (2010) 252303.

[9] CMS Collaboration, "Observation and studies of jet quenching in $\mathrm{PbPb}$ collisions at 2.76 TeV", Phys. Rev. C 84 (2011) 024906.

[10] CMS Collaboration, "Studies of jet quenching using isolated-photon+jet correlations in $\mathrm{PbPb}$ and pp collisions at $\sqrt{s_{N N}}=2.76 \mathrm{TeV}$ ", Phys. Lett. B 18 (2013) 773.

[11] ATLAS Collaboration, "Measurement of the correlation of jets with high $p_{T}$ isolated prompt photons in lead-lead collisions at $\sqrt{s_{N N}}=2.76 \mathrm{TeV}$ with the ATLAS detector at the LHC", (2012) ATLAS-CONF-2012-121.

[12] N. Armesto, B. Cole, C. Gale, W.A. Horowitz, P. Jacobs, et al., Phys. Rev. C 86 (2012) 064904.

[13] M. L. Miller, K. Reygers, S. J. Sanders, and P. Steinberg, "Glauber Modeling in High-Energy Nuclear Collisions ”, Annu.Rev. Nucl. Part. Sci. 57, 205 (2007).

[14] ATLAS Collaboration, "Measurement of the jet radius and transverse momentum dependence of inclusive jet suppression in lead-lead collisions at $\sqrt{s_{N N}}=2.76 \mathrm{TeV}$ with the ATLAS detector", Phys. Lett. B 719 (2013) 220-241.

[15] CMS Collaboration, "Measurement of the b-jet to inclusive jet ratio in $\mathrm{PbPb}$ and pp collisions at $\sqrt{s_{N N}}=2.76 \mathrm{TeV}$ with the CMS detector", CMS-PAS-HIN-12-003 (2012).

[16] Vitev, I. et. al.,"A theory of jet shapes and cross sections”, JHEP 0811:093, 2008.

[17] G. Salam, et. al., "The anti- $k_{T}$ jet clustering algorithm”, JHEP 04 (2008) 063.

[18] CMS Collaboration, "Particle-Flow Event Reconstruction in CMS and Performance for Jets, Taus, and $E_{T}^{\text {miss }}$, CMS-PAS-PFT-09-001 (2009).

[19] CMS Collaboration, "Detailed Characterization of Jets in Heavy Ion Collisions Using Jet Shapes and JetFragmentation Functions", (2012) CMS-PAS-HIN-12-013. 